\title{
Raman Fibre Laser Based Amplification in Long-haul/ Unrepeatered Coherent Transmission Systems
}

\author{
Mingming Tan ${ }^{1}$, Pawel Rosa ${ }^{2}$, Md Asif Iqbal ${ }^{1}$, Son Thai Le ${ }^{3}$, Ian. D. Phillips ${ }^{1}$, \\ Sergei K. Turitsyn ${ }^{1}$, and Paul Harper ${ }^{1}$ \\ ${ }^{1}$ Aston Institute of Photonic Technologies, Aston University, Aston Triangle, Birmingham, UK \\ ${ }^{2}$ The National Institute of Telecommunications, Warsaw, Poland \\ ${ }^{3}$ Nokia Bell Labs, Stuttgart, Germany \\ Tel: (0121) 204 3512,e-mail: m.tan1@aston.ac.uk
}

\begin{abstract}
The paper reviews the basic principles and recent advances of Raman fibre laser (RFL) based amplification techniques in long-haul/unrepeatered coherent transmission systems.

Different aspects of RFL based amplification have been characterised, including signal power distributions and relative intensity noise (RIN). Various RFL based amplifier designs have been evaluated in long-haul coherent transmission systems. The results shows the Fabry-Perot fibre laser based amplifier with two fibre Bragg gratings (FBGs) gives significant Q factor penalty using symmetrical bidirectional pumping, as the RIN of the signal is increased dramatically. However, random distributed feedback fibre laser based amplifier with a single FBG near the output section can mitigate the RIN of the signal, which enables the use of bidirectional second order pumping and therefore gives the best transmission performance up to $7915 \mathrm{~km}$. Furthermore, using random DFB fibre laser based amplifier has been proven to be effective to combat nonlinear impairment. In addition, unrepeatered transmission over $>350 \mathrm{~km}$ fibre length using RFL based amplification technique has been demonstrated experimentally using DP-QPSK WDM signals.
\end{abstract}

Keywords: Raman amplification, coherent fibre optic communications, Raman fibre laser.

\section{INTRODUCTION}

Distributed Raman amplification (DRA) compensates the fibre attenuation along the transmission span, which creates the amplification significantly different from using lumped Erbium-doped fibre amplifier (EDFA) [1]. The main advantage of DRA is that the signal-to-noise ratio (SNR) can be improved because the signal power variation (SPV) along the span is minimised [2]. This is a crucial feature as advanced modulation format requires high SNR. To minimise the signal power variation, forward-propagated (bi-directional) and high order pumping schemes are necessary [3]. However, forward-propagated (FW) pumping typically introduces relative intensity noise (RIN) to the signal [4]. Due to the capability of RIN mitigation, second order Raman fibre laser (RFL) based amplification has been proven to be an excellent candidate in both long-haul and unrepeatered transmission [5-10]. This paper discusses the key technologies and recent advances in RFL based amplification, which mitigate the RIN penalty and extend the maximum reach in long-haul transmission. In addition, RFL based amplification also provides superior performance in unrepeatered transmission system and can be compatible to the use of remote optically pumped amplifier to further improve the transmission performance and amplification bandwidth.

\section{RAMAN FIBRE LASER BASED AMPLIFICATION TECHNIQUE}

In the Raman fibre laser based amplifier configuration, a matched pair of $\sim 95 \%$ reflectivity FBGs with a centre peak at $1455 \mathrm{~nm}$ and a $3 \mathrm{~dB}$ bandwidth of $\sim 0.5 \mathrm{~nm}$ were used located at each end of an $83 \mathrm{~km}$ SMF. Highly depolarised second-order pumps at $1366 \mathrm{~nm}$ with RIN level of approximately $-120 \mathrm{~dB} / \mathrm{Hz}$ were used to create a fibre laser at the FBG's peak wavelength. In [8,9], we illustrated that the fibre laser was random distributed feedback (DFB) when using only BW-pumping [11-13], and Fabry-Perot laser when using bi-directional pumping (configuration R1 in Fig. 1(a)). If the FBG near the input side was removed, the fibre laser would be random DFB laser for both BW-pumping only and bidirectional pumping (R2 in Fig. 1(b)) [9,14]. Fig.1(c) and 1(d) shows signal power profiles along the fibre using the scheme R1 and R2, respectively. It can be seen that as the FW pump power increased, the signal power variations became smaller using both schemes. Using scheme R1 with $46.4 \% \mathrm{FW}$ pump power ratio to the total power, the power variation was minimised to only $1.6 \mathrm{~dB}$. In comparison, the variation was decreased to $4 \mathrm{~dB}$ using scheme R2. From the pump power tables shown in Fig. 1, we may notice that the pump power requirement in bidirectional configurations was lowest using scheme R1. However, the benefit of random DFB lasing in scheme R2 is that the cavity length of a random laser was distributed with a much shorter upper limit $(30-40 \mathrm{~km})$ compared to a Fabry-Perot cavity laser [12,13]. This means the RIN transfer could be reduced due to this shorter/distributed cavity, as shown in Fig. 1(e) and 1(f). Using the scheme R1, the RIN of the signal increased significantly as the FW-pump power increased, but the signal RIN remained the same using scheme R2. Further investigations on the impact of signal RIN with different FBG reflectivities near the input end can be found in $[15,16]$. In addition, other works to minimise the 
RIN penalty has focused on using first order FW pump instead of second order pump with FBG, because the use of first order pump source can have significantly higher Raman gain efficiency and require much lower pump power [17-19].
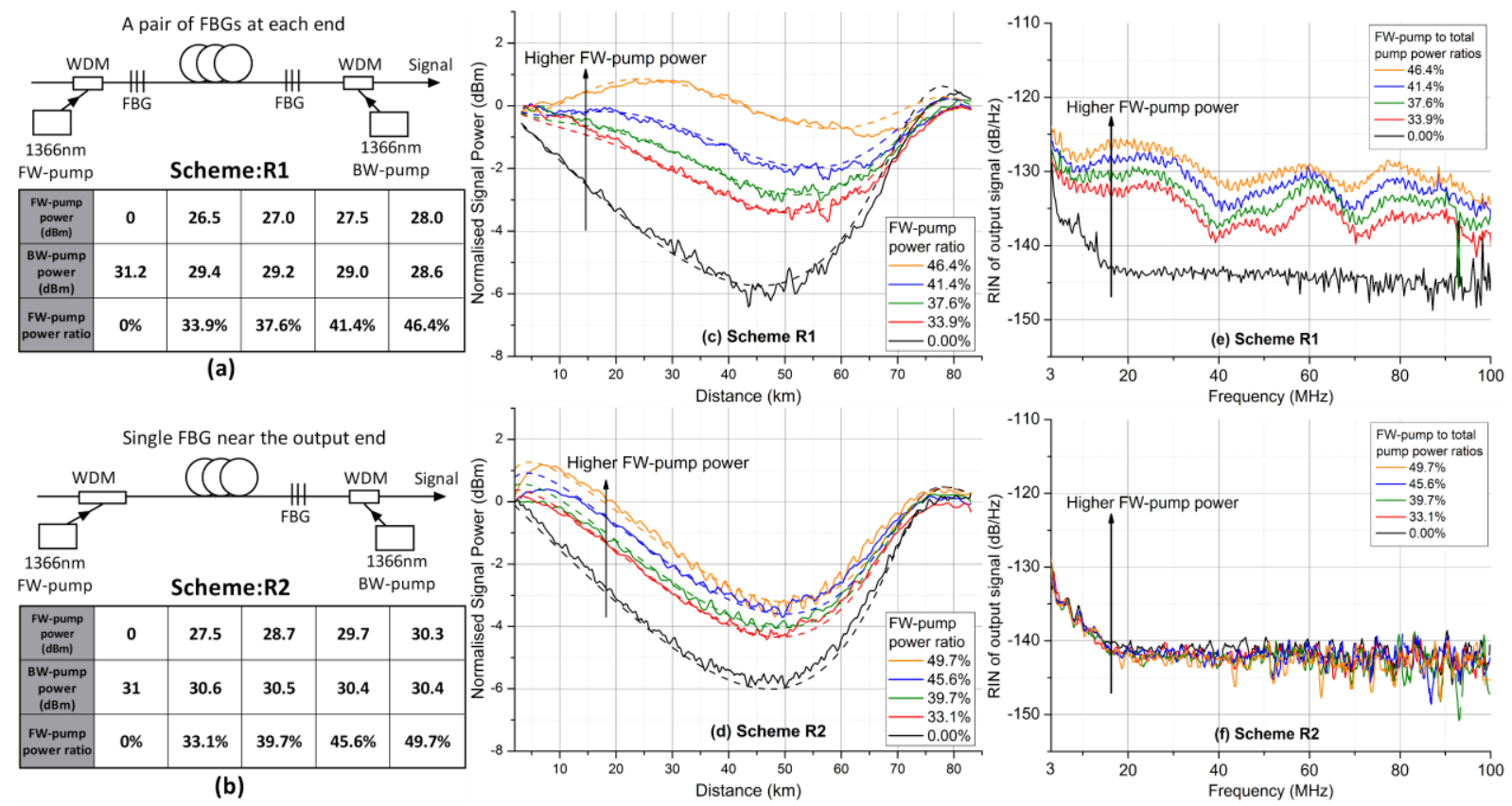

Figure 1. (a). Scheme R1: Raman fibre laser based amplifications with two FBGs.(b) Scheme R2: Raman fibre

laser based amplification with single FBG near the output. (c). Signal power profiles along the fibre using scheme Rl with different pump powers. (d). Signal power profiles along the fibre using scheme R2 with different pump powers. (e). RIN of the signal using scheme Rl with different pump powers. (f). RIN of the signal using scheme 22 with different pump powers.

\section{RFL BASED AMPLIFICATION IN LONG-HAUL REPEATERED TRANSMISSION}

To evaluate different RFL based amplifier schemes, a long-haul recirculating loop experiment was conducted using the setup shown in Fig. 2(a). The test signals consisted of ten $120 \mathrm{~Gb} / \mathrm{s}$ DP-QPSK channels with $100 \mathrm{GHz}$ spacing. The transmission span in the recirculating loop was $83.32 \mathrm{~km}$ standard single mode fibre with a total loss of $\sim 17.6 \mathrm{~dB}$ including $16.5 \mathrm{~dB}$ from SMF-28 fibre and $1.1 \mathrm{~dB}$ from WDM couplers. The AOM, $3 \mathrm{~dB}$ coupler, gain flattening filter (GFF), and WDMs gave a total loss of $\sim 12 \mathrm{~dB}$, which was compensated by an EDFA at the end of the loop. The receiver was a standard coherent detection setup and digital signal processing (DSP) was used offline with standard algorithms. Q factors were calculated from bit wise error rates.

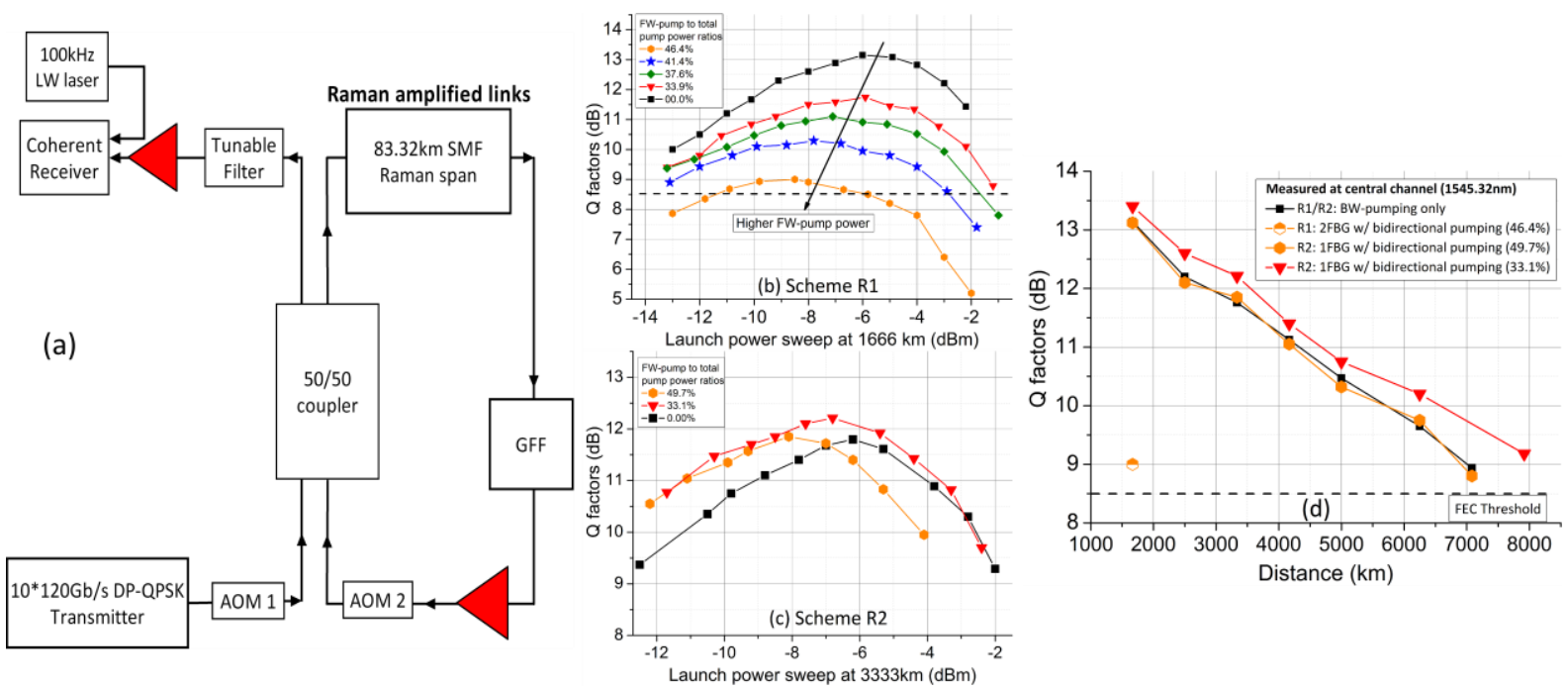

Figure 2. (a). Schematic diagram of long-haul repeatered transmission. (b). Q factors vs signal launch power using scheme R1. (c). Q factors vs signal launch powers using scheme R2. (d). Q factors vs transmission distances using scheme R1 or R2. 
When scheme R1 was used in the long-haul transmission, as shown in Fig. 1(b), the measured Q factor at $1666 \mathrm{~km}$ decreased from $13.1 \mathrm{~dB}$ (BW-pumping only) to $9 \mathrm{~dB}$ (symmetric bidirectional pumping) as the FWpumping power was increased. This result clearly demonstrated that using higher FW-pumping power introduced an overall $\mathrm{Q}$ factor penalty, despite the reduction in amplifier noise figure due to the use of FW-pump [8]. On the other hand, there was also a reduction in the optimum launch power as the FW-pump power was increased. This is due to the more uniform power level - for the same launch power there is more nonlinear penalty as FW-pump power is increased hence optimum launch power is decreased. Here, it should be noted that using higher FW-pump power degraded the system performance even with relatively low launch power per channel (i.e. $-13 \mathrm{dBm}$ ), when the impact of fibre nonlinearity was negligible. This means that the increase of nonlinearity was not responsible for the performance degradation, when the FW-pump power was increased. However, in scheme R2 (Fig. 2(c)) with the FBG near the input removed, the Q factor at 3333km with FW pump power ratio of $33 \%$ was $0.6 \mathrm{~dB}$ better than BW-pumping only, and even using $\sim 50 \% \mathrm{FW}$ pump power ratio still had comparable Q factor to BW-pumping only. This means in this scheme, the RIN penalty introduced by FWpumping was minimised, which indicates the uniform distribution in the signal power profile and consequently lower ASE noise led to actual performance improvement in long-haul transmission. Fig. 2(d) shows the Q factors versus transmission distances using both schemes R1 and R2. It can be clearly seen that using scheme R2 had similar or better transmission performance (up to $7915 \mathrm{~km}$ maximum reach) than BW-pumping only scheme, but using scheme R1 had a significant penalty and symmetrical bidirectional pumping can transmit only $1666 \mathrm{~km}$. An important application of this RIN-penalty free Raman amplifier scheme R2 was in the nonlinearity mitigation using mid-link optical phase conjugation (OPC), because using the scheme had very symmetrical signal power profile and could maximise the benefit of nonlinearity compensation using mid-link OPC. The details of this work can be found in [20-22].

\section{RFL BASED AMPLIFICATION IN UNREPEATERED TRANSMISSION}

In unrepeatered transmissions, distributed Raman amplifications offer better noise performance resulting in higher OSNR, compared with EDFA [23-25]. By using higher order distributed Raman amplifications, the signal power variation can be reduced leading to highly uniform signal power distribution and better transmission performance. Here, based on the RFL based amplification techniques, the transmission performance using $100 \mathrm{G}$ DP-QPSK WDM signals over up to $352.8 \mathrm{~km}$ SMF has been shown without using remote optically pumped amplifier (ROPA) or any specialty fibre.
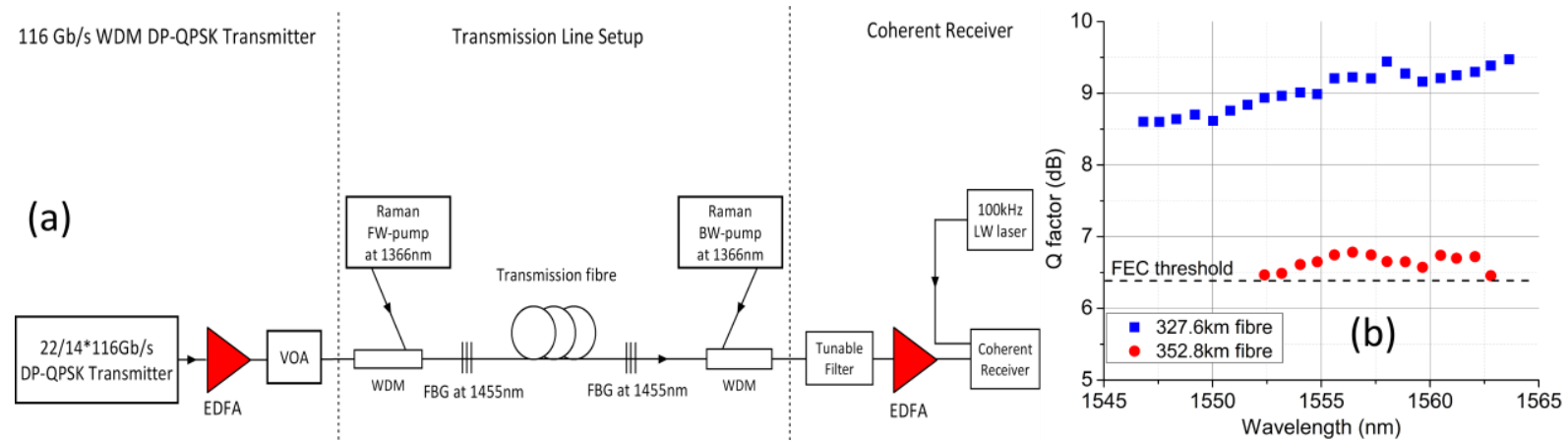

Figure 3. (a).Schematic diagram of Raman fibre laser based amplification in unrepeatered transmission. (b). $Q$ factors of all the measured channels at $327.6 \mathrm{~km}$ and $352.8 \mathrm{~km}$.

Fig. 3(a) shows the schematic of the unrepeatered transmission system using DP-QPSK WDM signals and RFL based amplification technique. An important difference of RFL amplifier in unrepeatered and repeatered system is that due to the fibre length of unrepeatered system (i.e. $300 \mathrm{~km}$ ), the generated Raman fibre laser at $1455 \mathrm{~nm}$ was no longer a Fabry-Perot fibre laser across the whole cavity/transmission fibre. Instead, it was actually two separate random DFB fibre laser located near each side of the span and had no interaction between each other [10]. Fig. 3(b) shows the $Q$ factors of all the measured channels at $327.6 \mathrm{~km}$ and $352.8 \mathrm{~km}$. At $327.6 \mathrm{~km}$, the maximum number of channels was limited to the number of lasers we had. At $352.8 \mathrm{~km}, 14$ channels were transmitted at the FEC threshold of $6.4 \mathrm{~dB}$. This was achieved without the use of ROPA and lowloss fibre, which indicates our proposed setup can be used to readily upgrade existing SSMF legacy link without installing new fibre. In addition, our proposed setup is also compatible with ROPA by simply adding the seed pump at $1480 \mathrm{~nm}$, which can further improve the transmission distance and amplification bandwidth.

\section{CONCLUSIONS}

In conclusion, different Raman fibre laser based amplification techniques have been characterised and evaluated in both long-haul repeatered and unrepeatered coherent transmission systems over SSMF. The results show that 
random DFB fibre laser based Raman amplification enables bidirectional second order pumping and thus offer better transmission performance, due to the mitigation of the signal RIN (thanks to the reduced efficiency of first order Stokes generation) and ASE noise. This scheme is also proved to be highly effective to compensate the nonlinear impairment and enhance the transmission distance in mid-link OPC transmission system. In addition, the scheme is highly flexible and adjustable to meet different requirement on the transmission links.

\section{ACKNOWLEDGEMENTS}

The work was funded by UK EPSRC programme grant UNLOC (EP/J017582/1), MSCA IF grant SIMFREE (No. 748767), and FP7 ITN programme ICONE (No. 608099). We thank Zhongyuan. Sun, Changle Wang, and Prof. Lin Zhang for providing the FBGs.

\section{REFERENCES}

[1] C. Headley, and G. P. Agrawal, "Raman amplification in fiber optic communication systems," Academic press, 1st edition, 2005.

[2] J. Bromage, "Raman Amplification for Fiber Communications Systems," J. Lightwave Technol., vol. 22 , pp. 79-93, 2004.

[3] J. -C. Bouteiller et al., "Quasi-constant signal power transmission,” in ECOC 2002, 1-2.

[4] C. R. S. Fludger et al., "Pump to signal RIN transfer in Raman fiber amplifiers," J. Lightwave Technol. Vol. 19, pp. 1140-1148, 2001.

[5] J. D. Ania-Castañón, "Quasi-lossless transmission using second-order Raman amplification and fiber Bragg gratings,” Opt. Express, Vol. 12, pp. 4372-4377, 2004.

[6] J. D. Ania-Castañón et al., "Ultralong Raman fiber lasers as virtually lossless optical media," Phys. Rev. Lett. vol. 96, 023902, 2006.

[7] J. D. Ania-Castañón et al, "Simultaneous Spatial and Spectral Transparency in Ultralong Fiber Lasers," Phys. Rev. Lett. vol. 101, 123903, 2008.

[8] M. Tan et al., "Evaluation of 100G DP-QPSK long-haul transmission performance using second order copumped Raman laser based amplification," Opt. Express vol. 23, pp. 22181-22189, 2015.

[9] M. Tan et al., "Transmission performance improvement using random DFB laser based Raman amplification and bidirectional second-order pumping," Opt. Express, vol. 24, pp. 2215-2221, 2016.

[10] P. Rosa et al., "Unrepeatered DP- QPSK transmission over 352.8 km SMF using random DFB fiber laser amplification” IEEE Photon. Technol. Lett. Vol. 27, pp. 1041-1135, 2015.

[11] S. B. Papernyi et al., "Third-order cascaded Raman amplification," in OFC 2002, paper FB4.

[12] D. V. Churkin et al., "Raman fiber lasers with a random distributed feedback based on Rayleigh scattering," Phys. Rev. A, vol. 82, 033828, 2010.

[13] S. K. Turitsyn et al., "Random distributed feedback fibre laser," Nat. Photonics, vol. 4, 231-235 (2010).

[14] M. Tan et al., "Extended Reach of 116 Gb/s DP-QPSK Transmission using Random DFB Fiber Laser Based Raman Amplification and Bidirectional Second-order Pumping," in OFC 2015, paper W4E.1.

[15] M. Tan et al., "RIN mitigation in second- order pumped Raman fibre laser based amplification," in ACP 2015, paper AM2E.6.

[16] G. Rizzelli et al., "Impact of input FBG reflectivity and forward pump power on RIN transfer in ultralong Raman laser amplifiers," Opt. Express, vol. 24, pp. 29170-29175, 2016.

[17] M. Tan et al. "RIN-Penalty Mitigation And Transmission Performance Improvement Using ForwardPropagated Broadband First Order Raman Pump," accepted, in OECC, 2017.

[18] M. Tan et al., "Evaluation of Long-haul Coherent Transmission Performance Using Low RIN Forward Raman Pump," in ACP 2016, paper AF3D.2.

[19] V. Dvoyrin et al., "Bi-doped Fiber Laser for Telecom Applications," in CLEO 2016, paper STu1F.4.

[20] A. D. Ellis et al., "4 Tbit/s transmission reach enhancement using 10x400 Gbit/s super-channels and polarization insensitive dual band optical phase conjugation," J. Lightwave Technol., Vol.34, pp. 17171723, 2016.

[21] I. D. Phillips et al., "Exceeding the Nonlinear-Shannon limit using Raman laser based amplification and optical phase conjugation," in OFC 2014, paper M3C.1

[22] P. Rosa et al., "Signal power asymmetry optimisation for optical phase conjugation using Raman amplification," Opt. Express, Vol. 23, pp. 31772-31778, 2015.

[23] W. S. Pelouch, "Raman Amplification: an Enabling Technology for High-Capacity, Long-Haul Transmission," in OFC 2015, paper. W1C.1.

[24] L. Galdino et al., "Amplification Schemes and Multi-Channel DBP for Unrepeatered Transmission," J. Lightwave Technol, Vol. 34, pp. 2021-2027, 2016.

[25] L. Galdino et al., "Unrepeatered Nyquist-PDM-16QAM transmission over 364km using Raman amplification and mulit-channel DBP," Opt. Lett., Vol. 40, pp. 3025-3028, 2015. 